\title{
Arsenic fate under the shadow of sea-level rise
}

Fatemeh Izaditame, Matthew G. Siebecker, Ryan Tappero, Piyapas Sricharoenvech and Donald L. Sparks 
- Sea level is on the rise due to global warming.

- $0.8 \mathrm{~m}$ by 2100 (IPCC, 2013).

- Global Mean sea level rise is about $3 \mathrm{~mm} \mathrm{yr}^{-1}$ (IPCC, 2013).

- SLR in Mid-Atlantic higher than anywhere else (Sallenger et al., 2012).

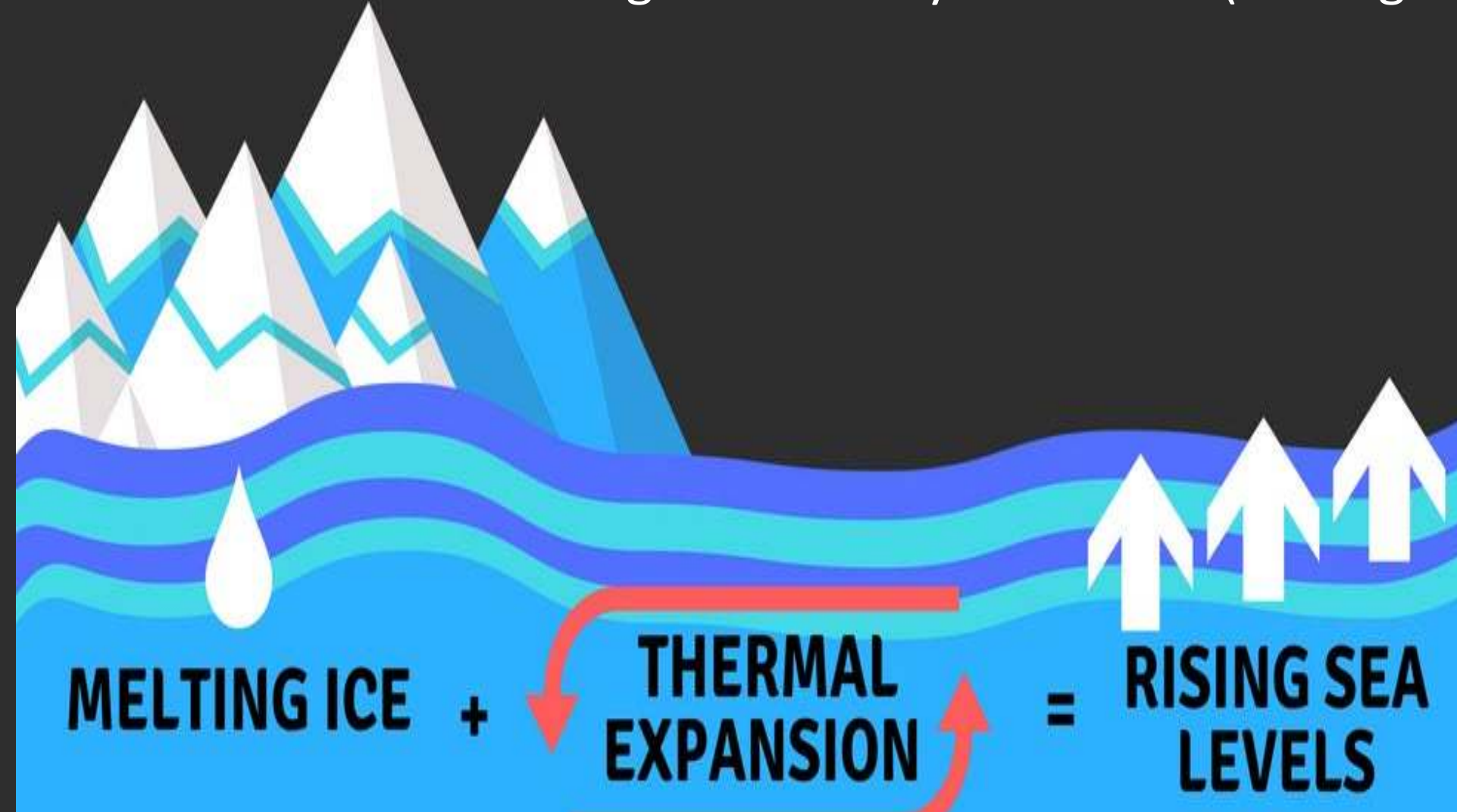




\section{Sea Level Rise \\ Population Impact}

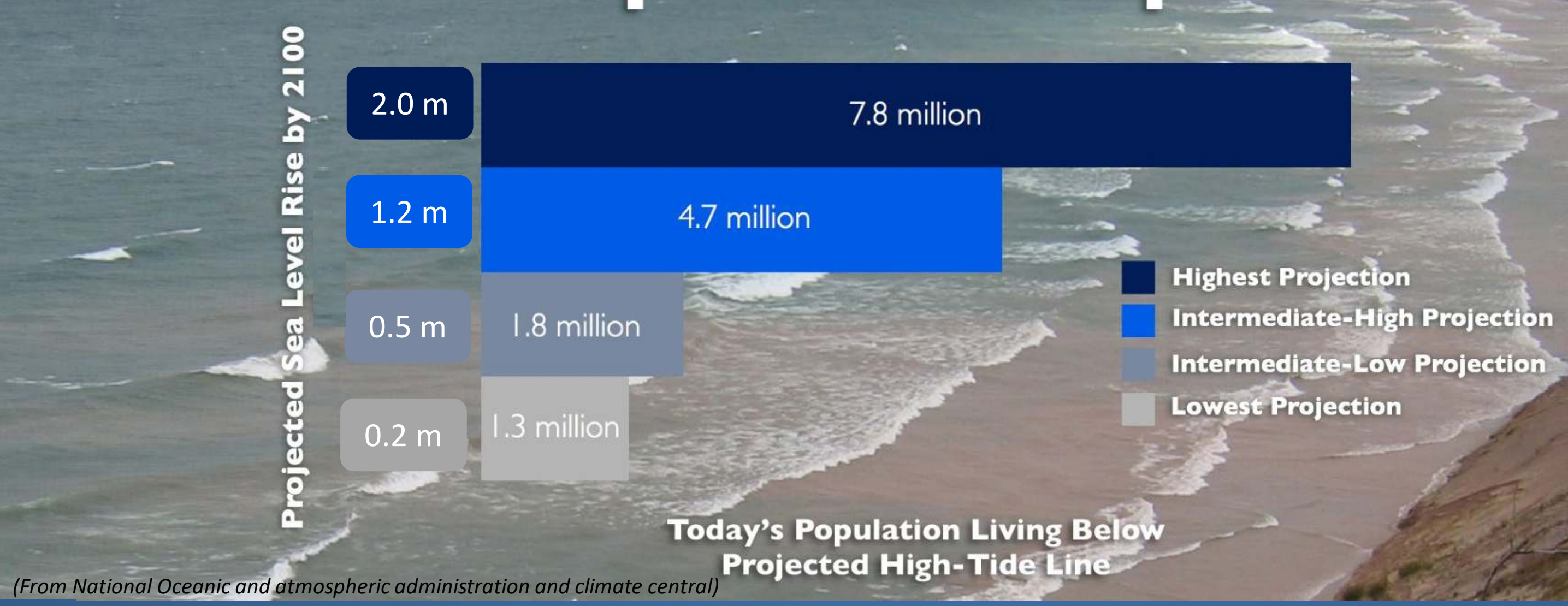

$44 \%$ of the world's (40\% of the U.S. ) population lives along coasts (UN, 2010).

By 2100, SLR can be as high as 2 meters above 1992 levels.

Would put the homes of 7.8 million Americans at risk of being flooded. 


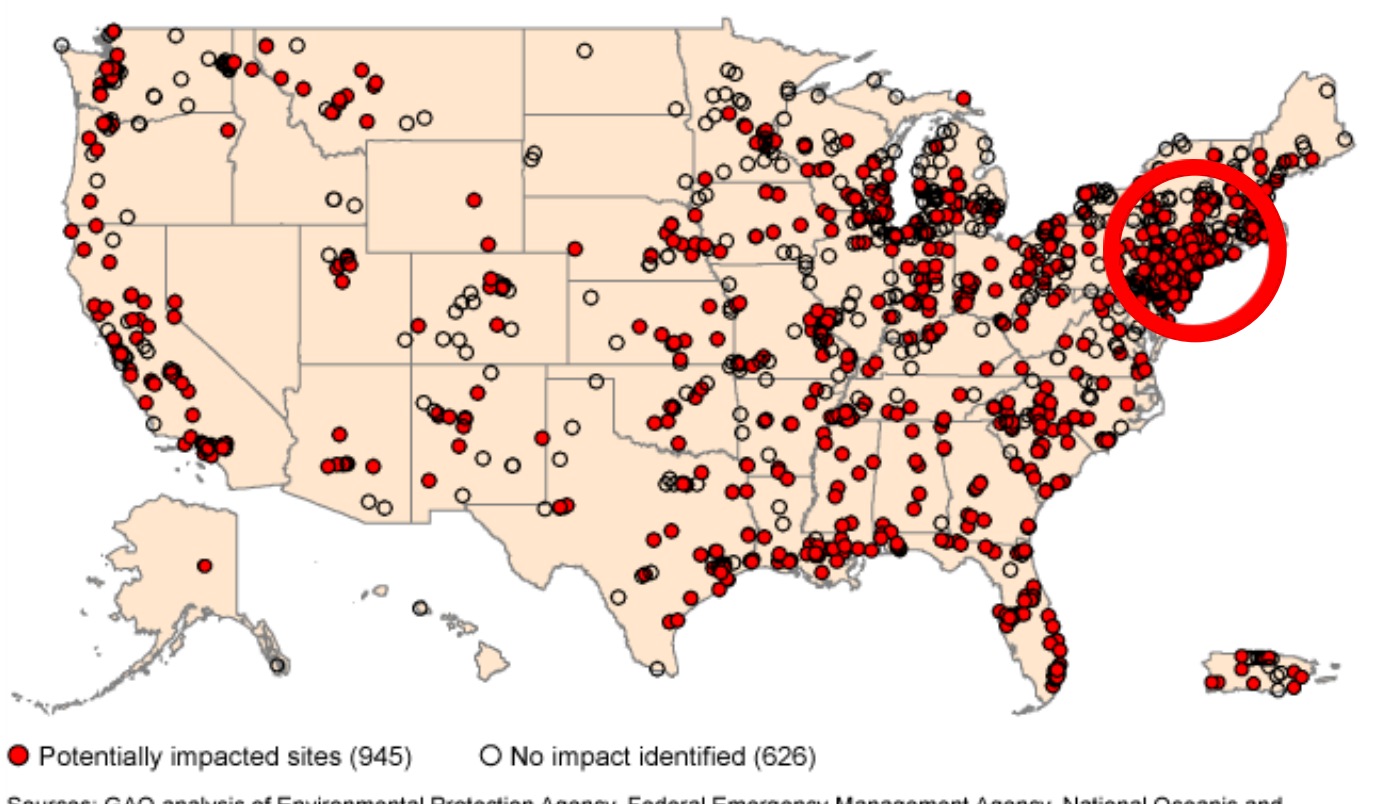

Sources: GAO analysis of Environmental Protection Agency, Federal Emergency Management Agency, National Oceanic and
Atmospheric Administration, and U.S. Forest Service data; Maplnfo (map). | GAO-20-73

Federal data suggests about 60 percent of Superfund sites overseen by EPA are in areas that may be impacted by wildfires and different types of flooding - natural hazards that may be exacerbated by climate change. (US GAO NOV 2019)

14 Philadelphia-area: Superfund sites are in flood zones and SLR areas

2,500 chemical sites are in the water's path

\section{How Rising Seas Will Affect Norfolk Base}

This animation shows the flooding that's likely to occur at Naval

Station Norfolk, at current and higher sea levels, during the type of

storm that hits on average once a year.

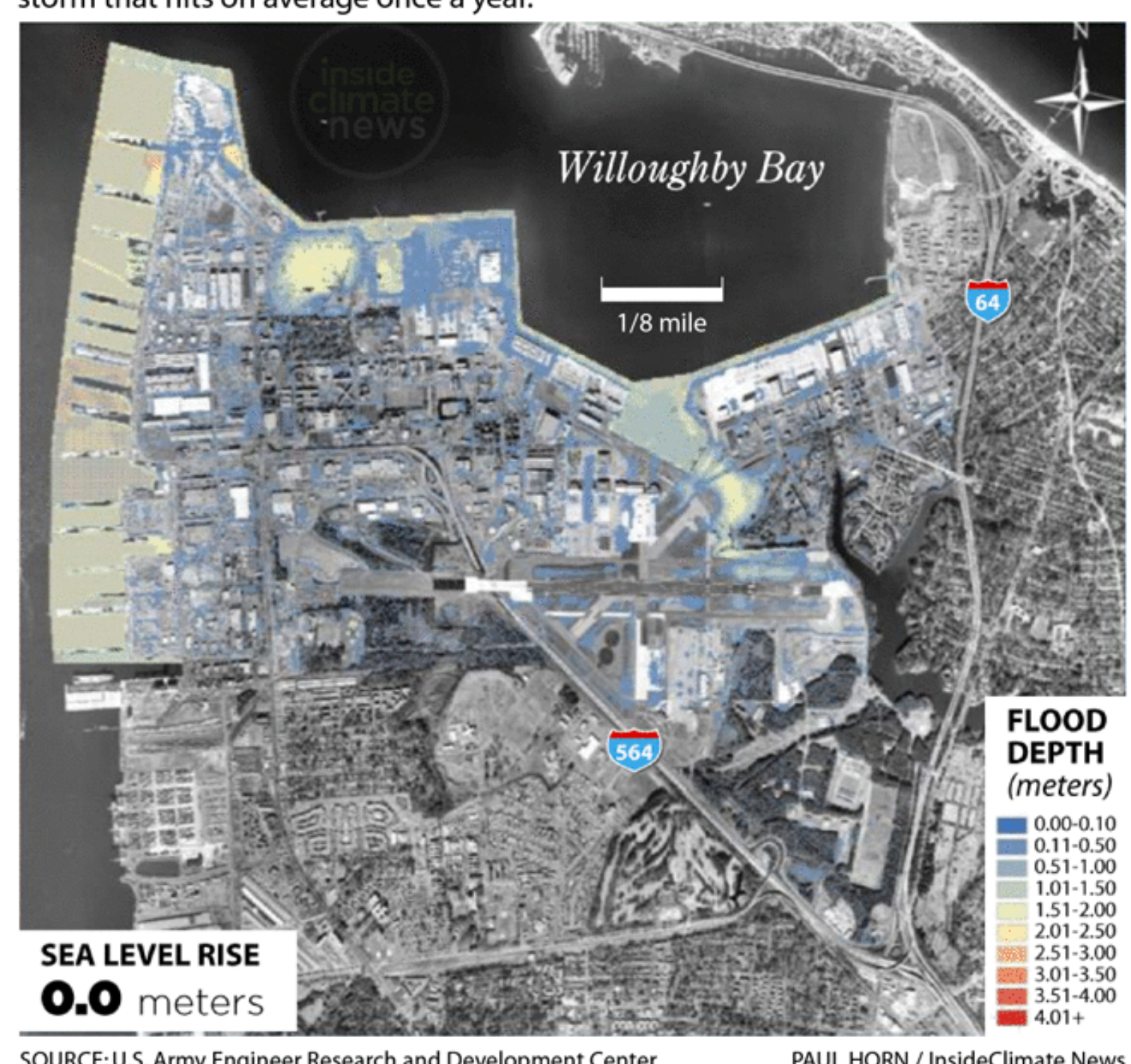

SOURCE:U.S. Army Engineer Research and Development Center 


\section{Study Location}
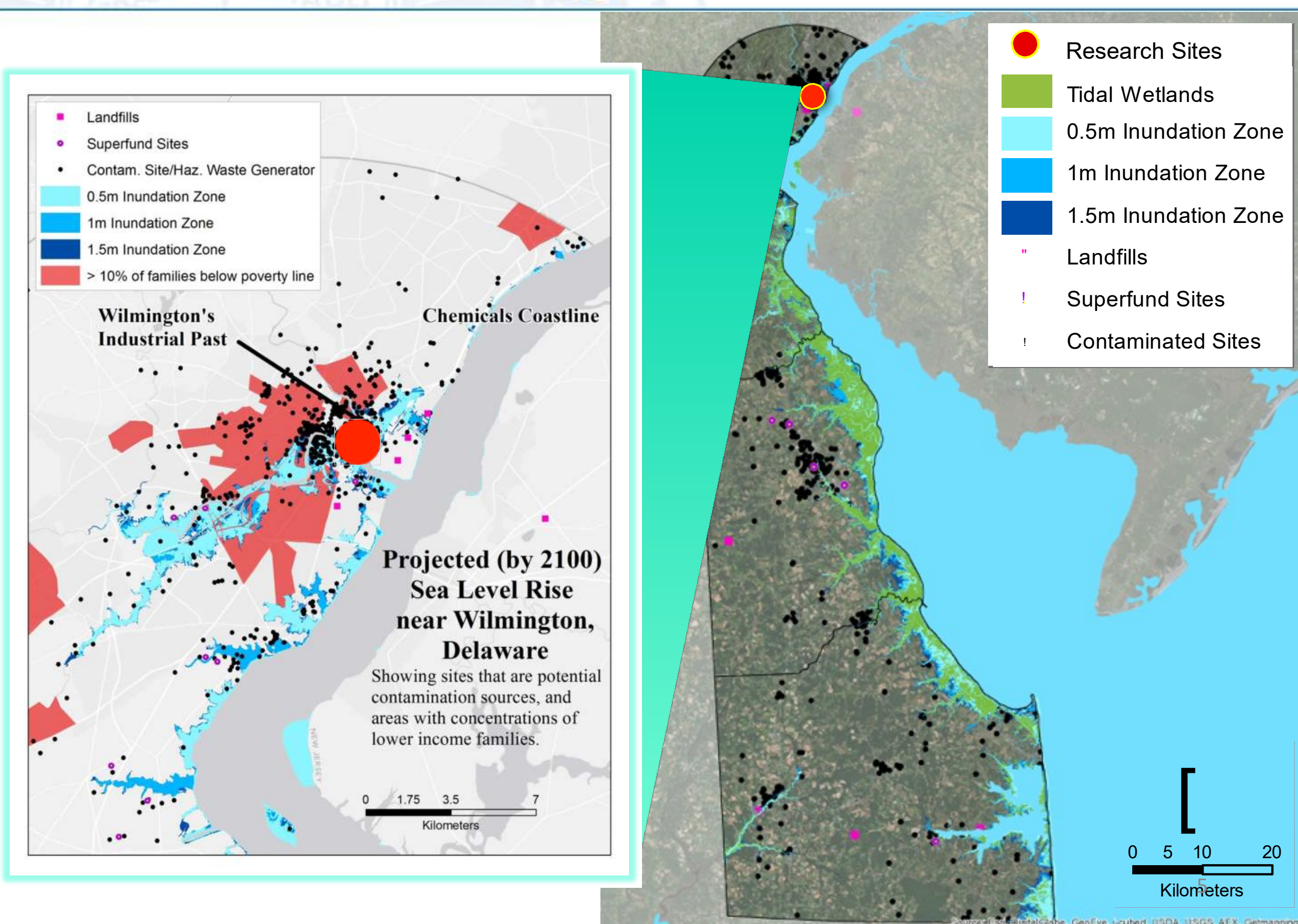


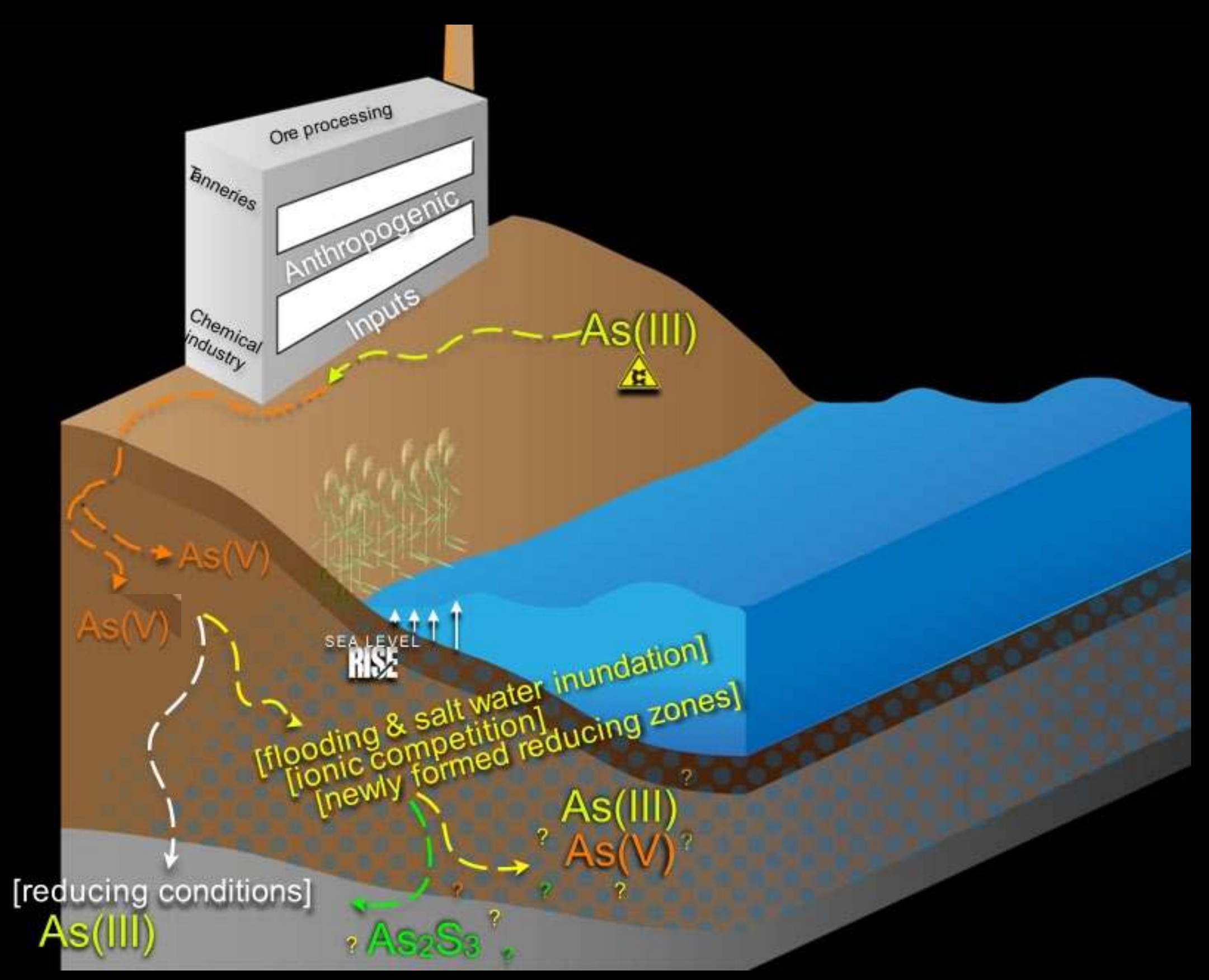

How will saltwater flooding impact cycling, mobility, and speciation of As in historically contaminated sediments? 
- Mixing of Porewater

- Oxidation of particles

- Sediment: water

- Salinity

Laminar Flow Condition
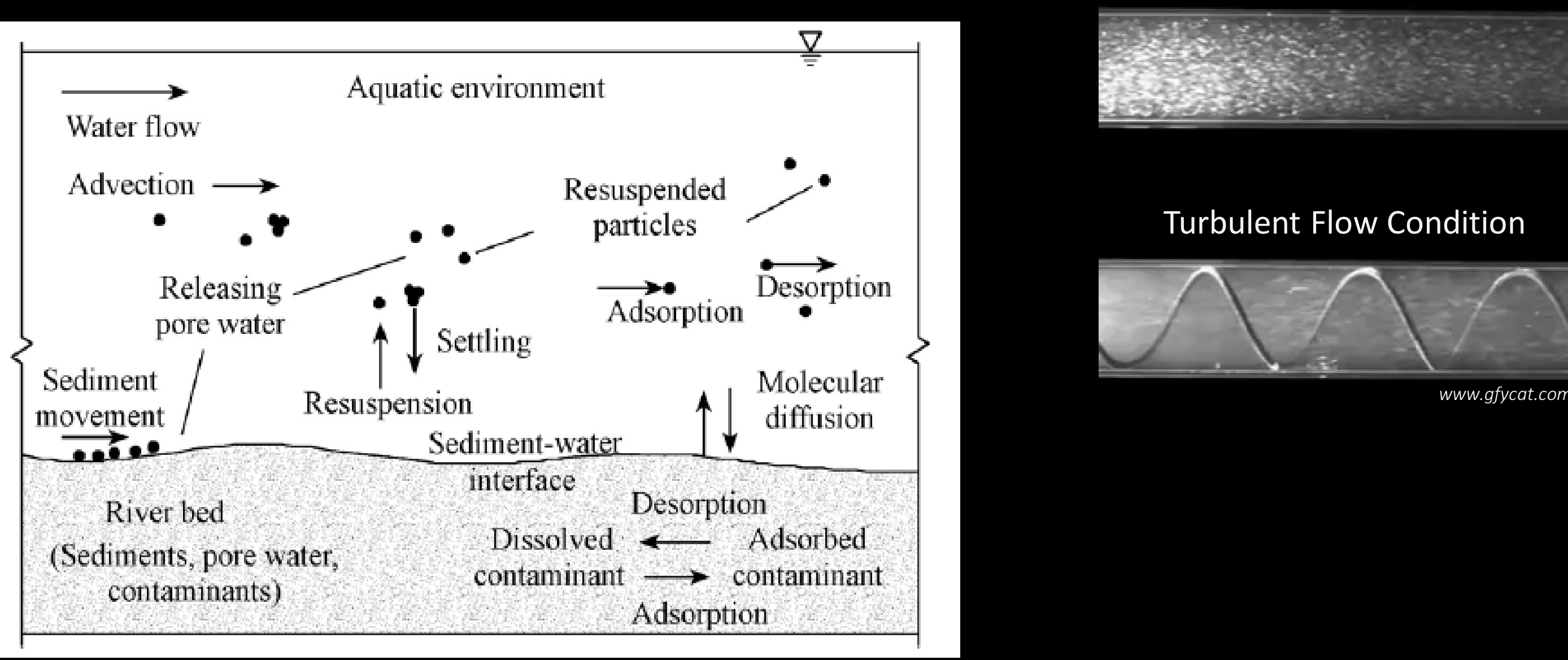

Turbulent Flow Condition

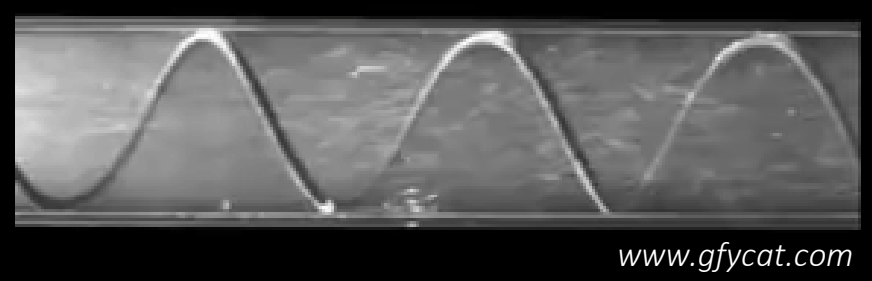




\section{Materials \& Methods}

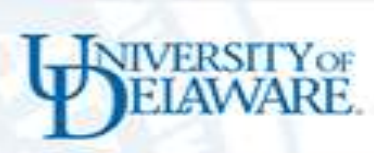

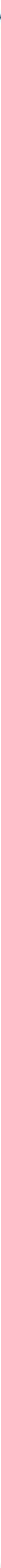




\section{Materials \& Methods}
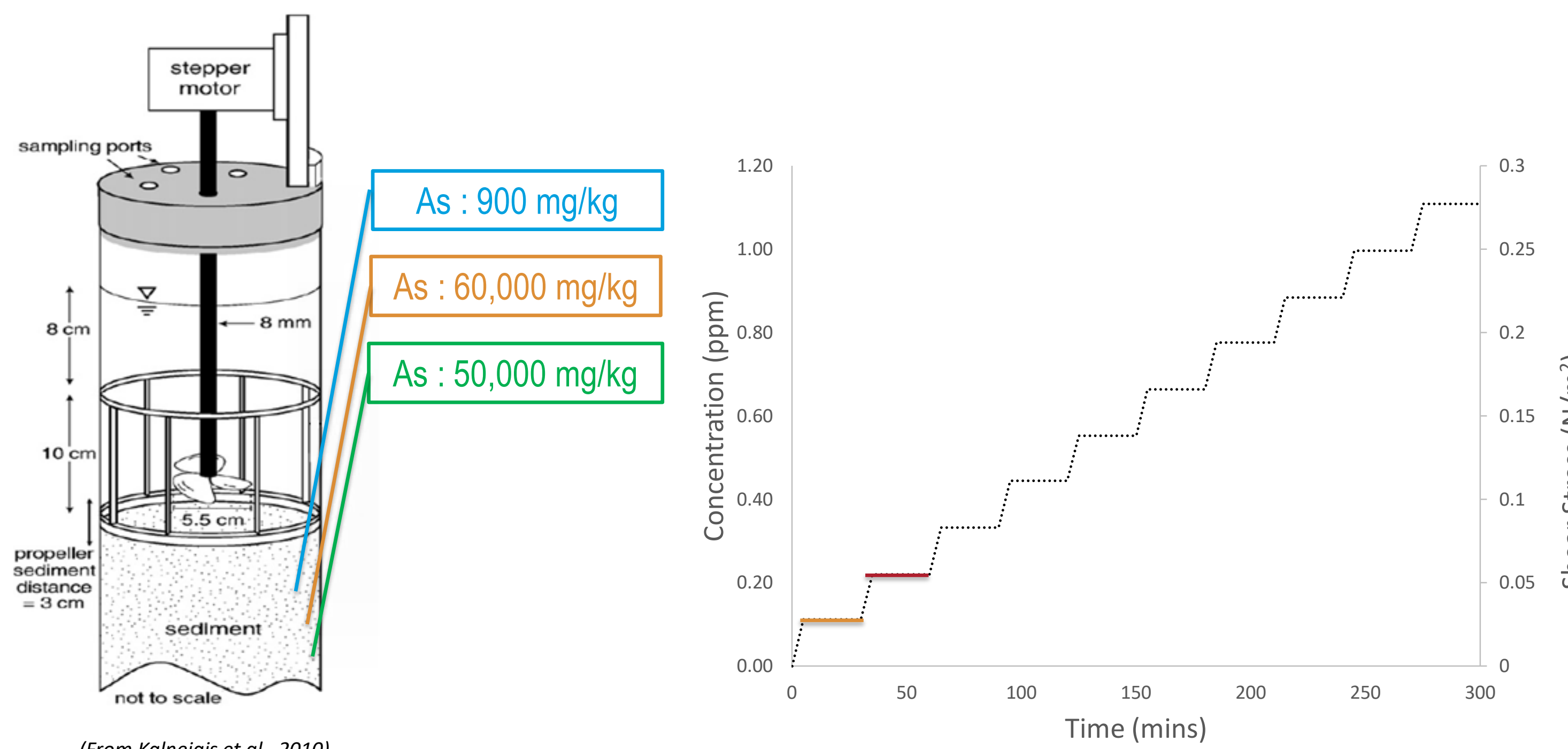

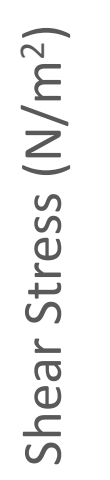

(From Kalnejais et al., 2010) 


\section{Arsenic release depends on initial As concentration \& shear stress}

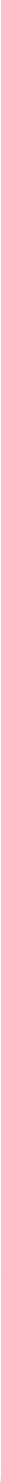




\section{Arsenic Speciation}

$140 \square$ Test 1 Test $2 \square$ Test 3

As : $900 \mathrm{mg} / \mathrm{kg}$

120

As : $60,000 \mathrm{mg} / \mathrm{kg}$

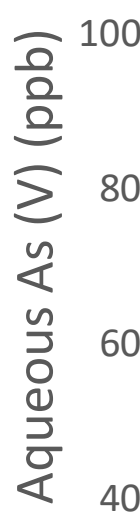

As : $50,000 \mathrm{mg} / \mathrm{kg}$

20

0
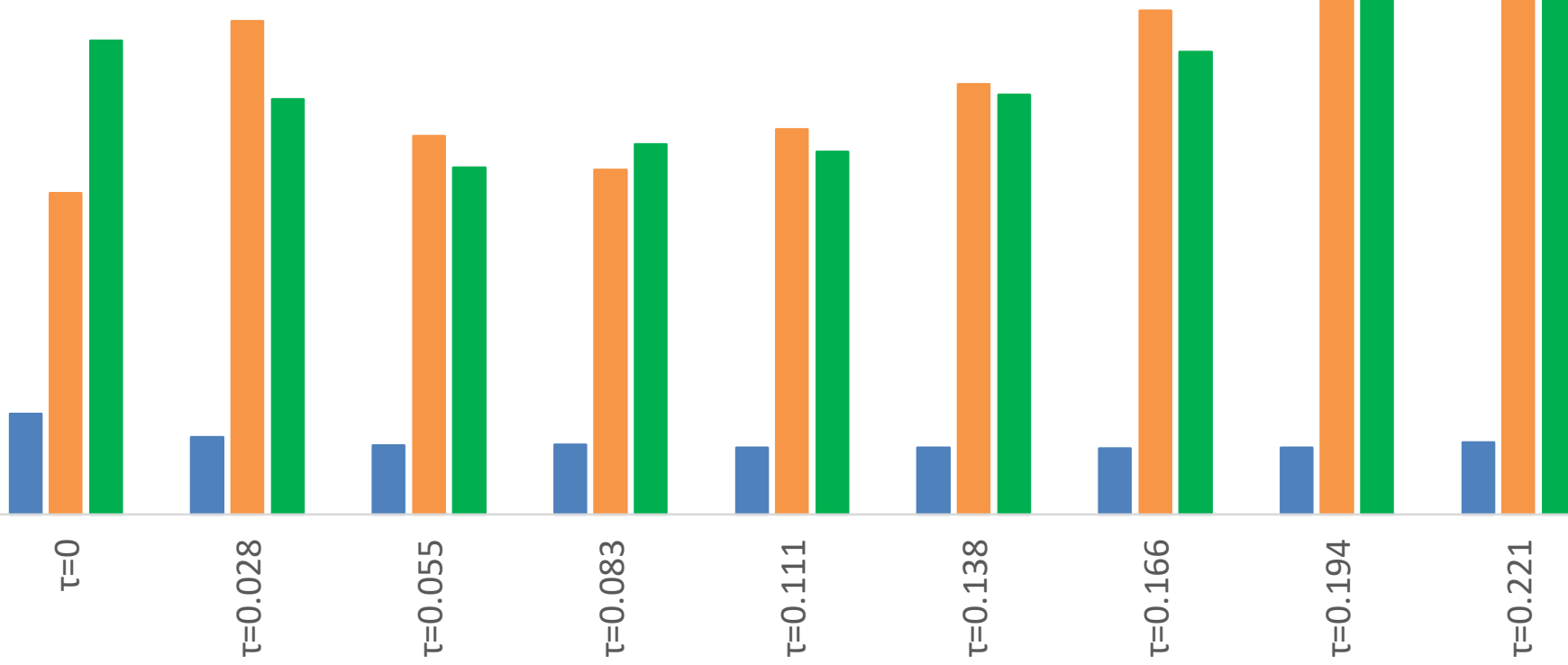

Shear Stress $\left(\mathrm{N} / \mathrm{m}^{2}\right)$ 


\section{Solid Phase Speciation}

\section{Before Flooding}

\section{During Flooding}




\section{Solid Phase As Speciation}

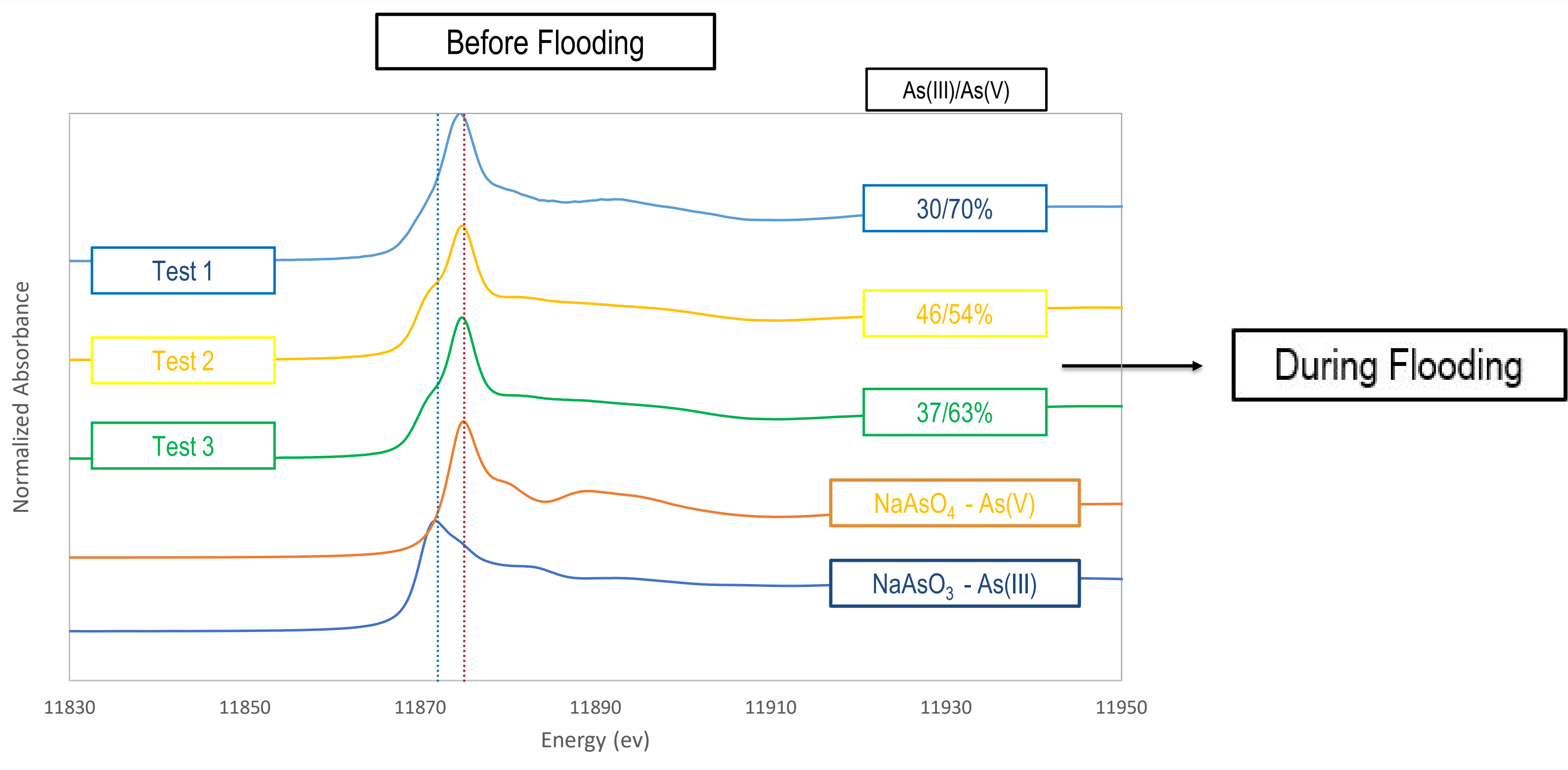




\section{Solid Phase As Speciation}

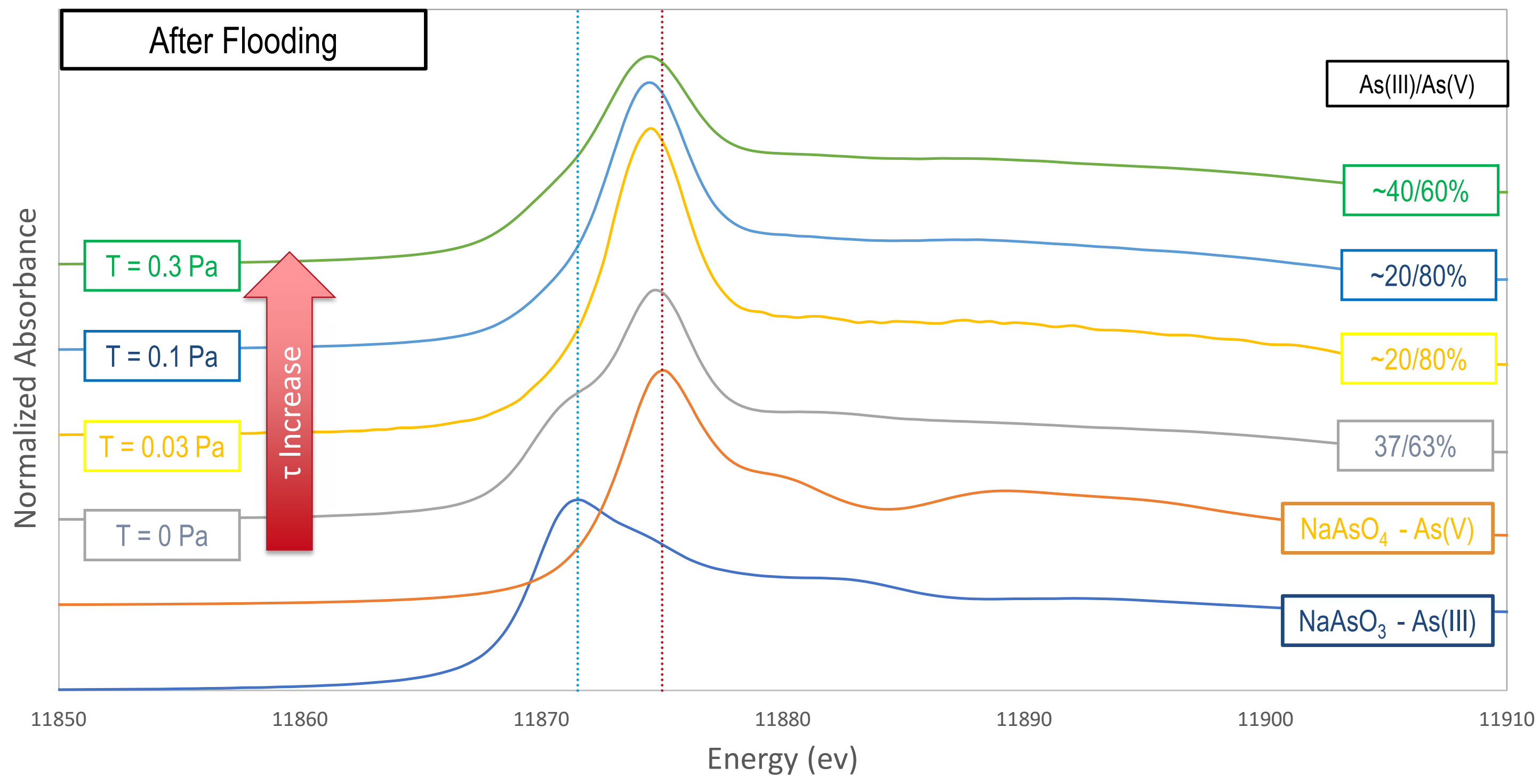




\section{As on suspended particles during flooding}

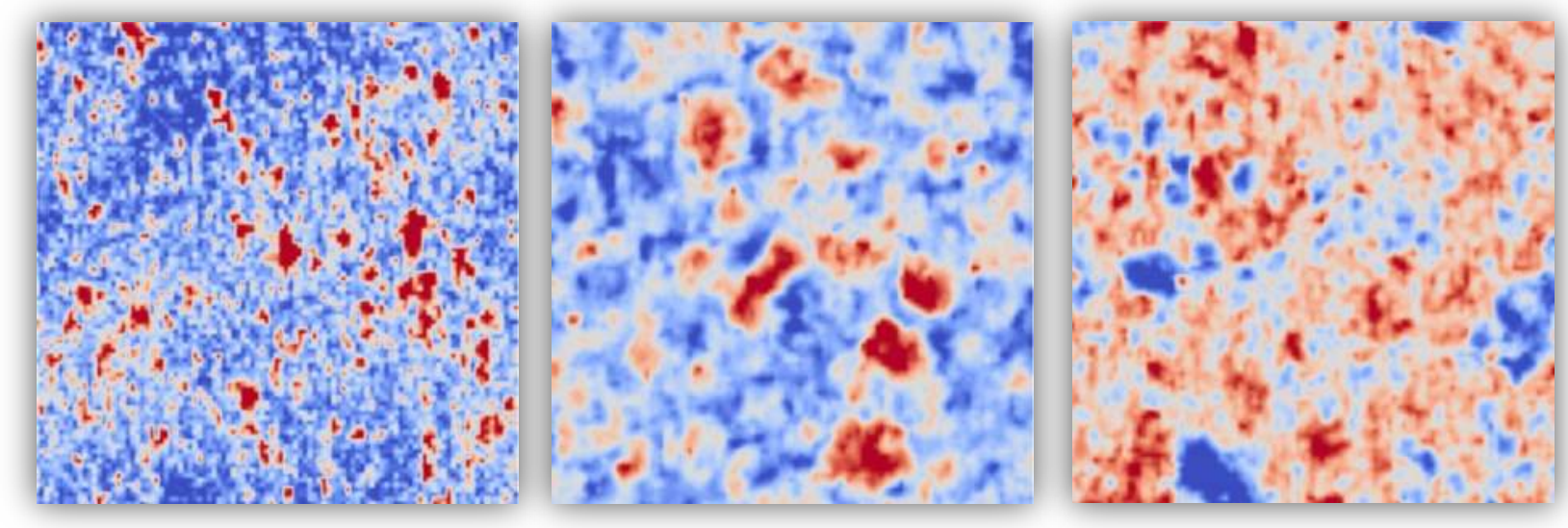

As $-\tau=0.028 \mathrm{~N} / \mathrm{m}^{2} \quad$ As $-\tau=0.11 \mathrm{~N} / \mathrm{m}^{2} \quad$ As $-\tau=0.28 \mathrm{~N} / \mathrm{m}^{2}$
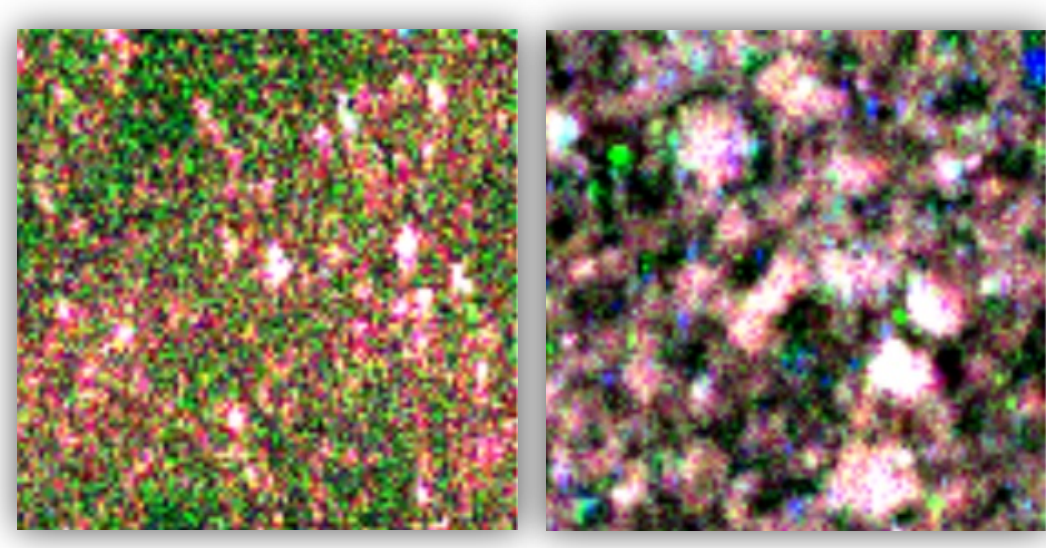

$$
\tau=0.028 \mathrm{~N} / \mathrm{m}^{2}
$$

$\tau=0.11 \mathrm{~N} / \mathrm{m}^{2}$

As-Ca-Zn

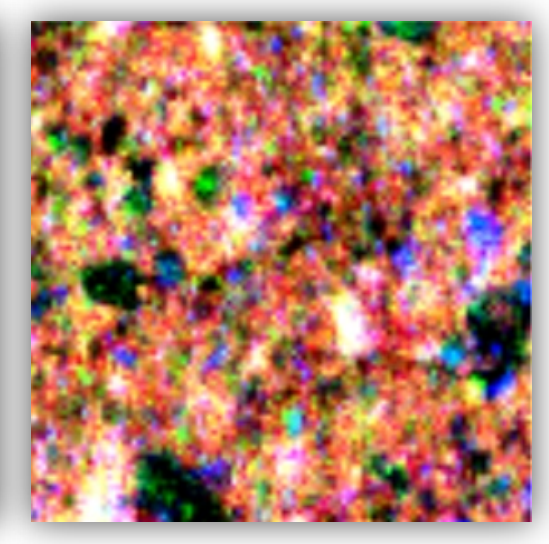

$\tau=0.28 \mathrm{~N} / \mathrm{m}^{2}$

More particles $\rightarrow$ More As

\begin{tabular}{|c|}
\hline Test 3 \\
\hline \hline As: \\
50,000 \\
$\mathrm{mg} / \mathrm{kg}$ \\
\hline
\end{tabular}

Co-location of $\mathrm{Ca}, \mathrm{As}, \mathrm{Fe}$, and $\mathrm{Zn}$ 


\section{Change in surface charge}

Flooding starts

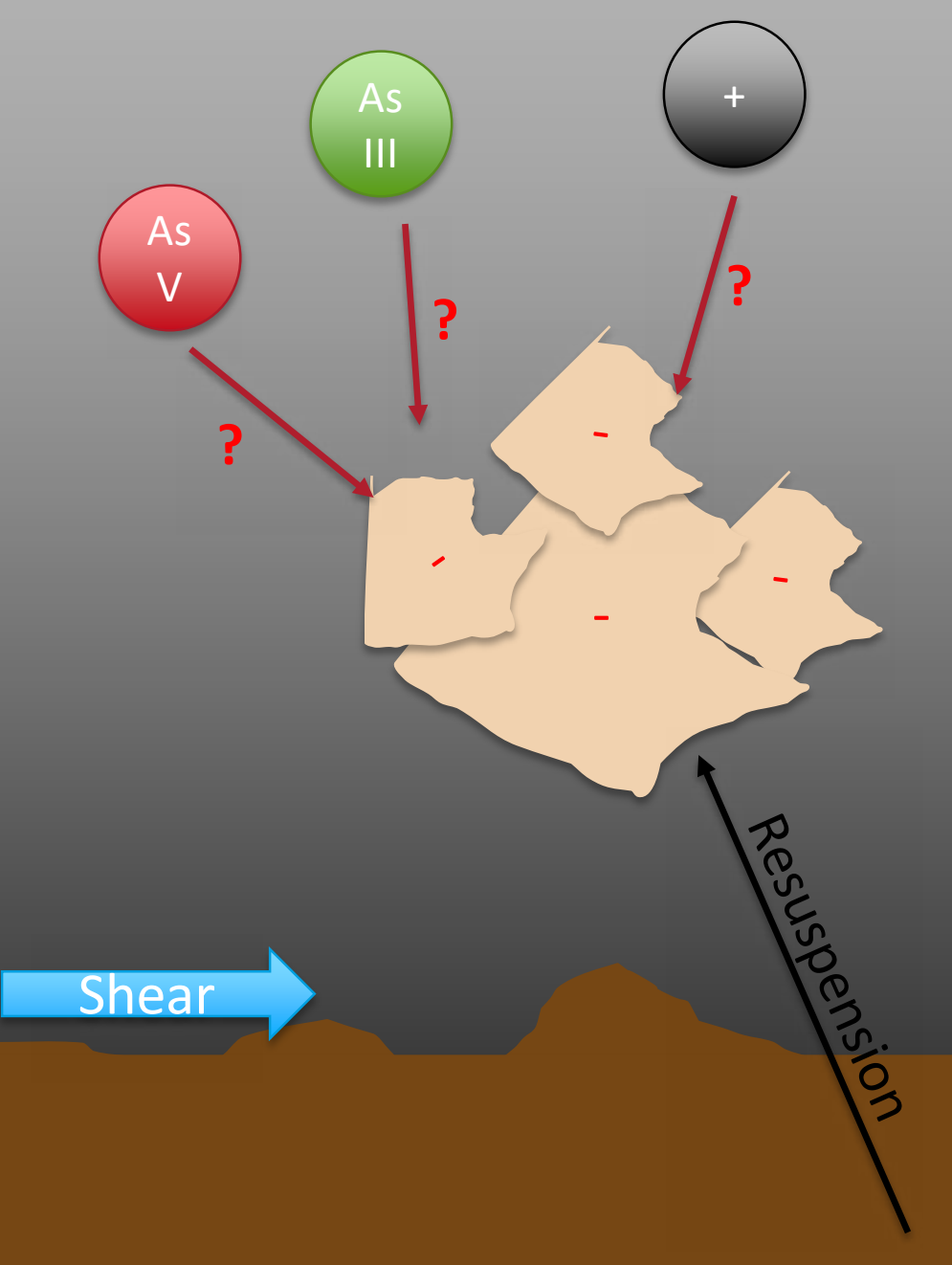

\section{As removal from the solution}

Low initial As in sediment

\section{Aqueous As accumulation}

High initial As in sediment

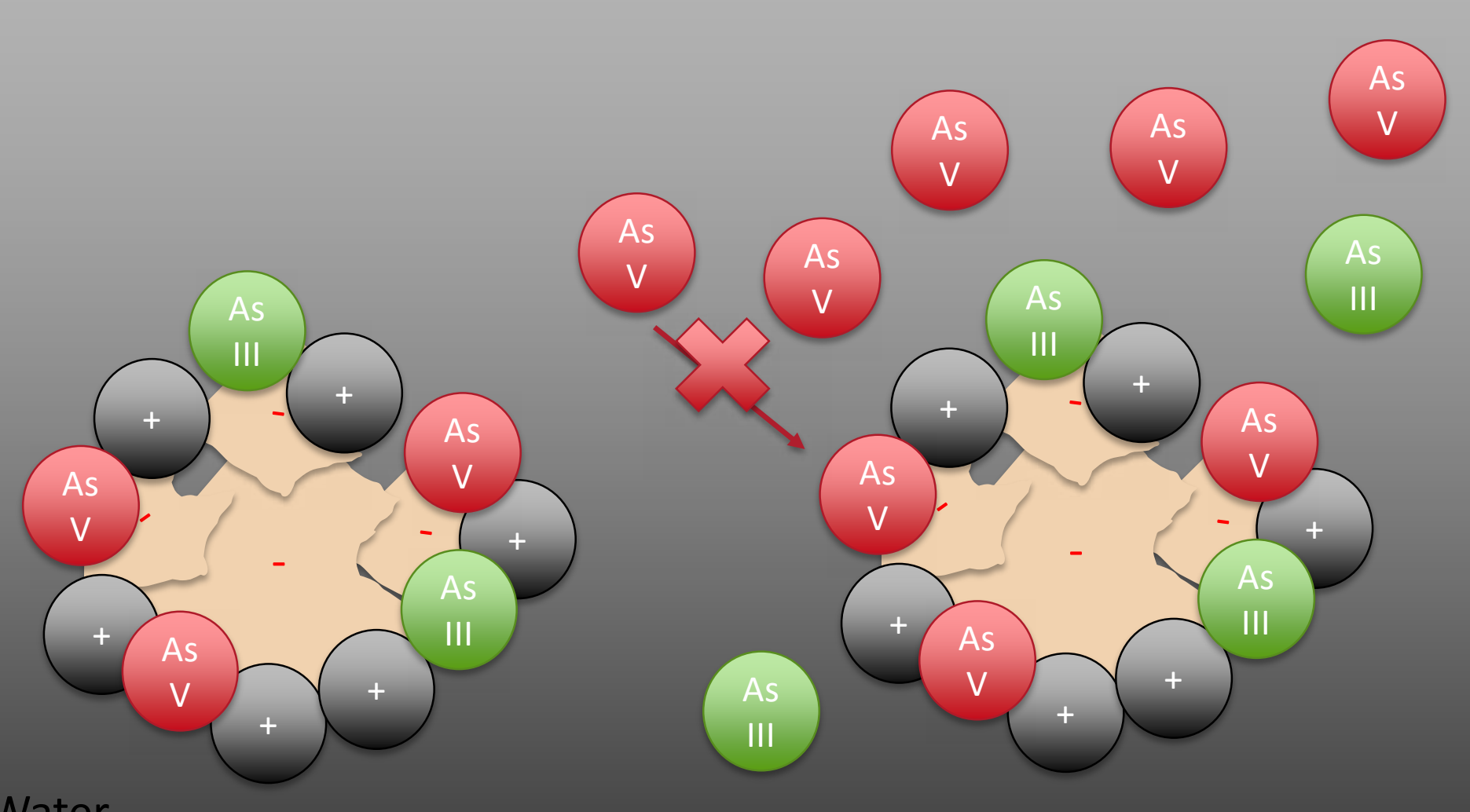

Overlying Water 


\section{Arsenic Speciation}

$140 \square$ Test $1 \square$ Test $2 \square$ Test 3

As : $900 \mathrm{mg} / \mathrm{kg}$

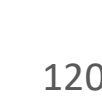

As : 60,000 mg/kg

120

100

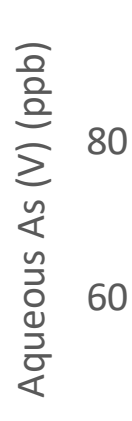

40

20

0

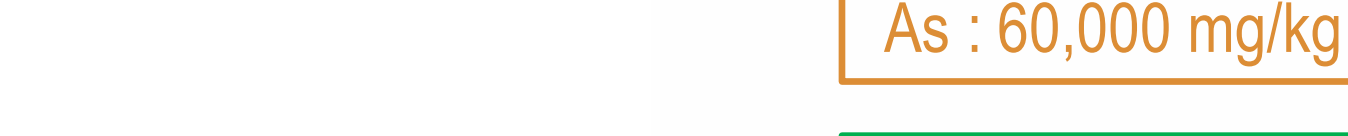

As : $50,000 \mathrm{mg} / \mathrm{kg}$

$\stackrel{11}{\mapsto}$

II

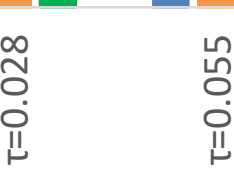

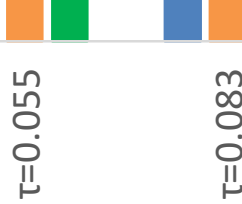

m

Shear Stress $\left(\mathrm{N} / \mathrm{m}^{2}\right)$ 


\section{Summary}

- SLR-induced flooding can increase As concentration in the solution.

- Released aqueous arsenic during the flooding was mainly As(V).

- Low intensity flooding can oxidize As on resuspended particles.

- Arsenic was mainly co-located with Fe, $\mathrm{Ca}$, and $\mathrm{Zn}$ before, during, and after flooding. 


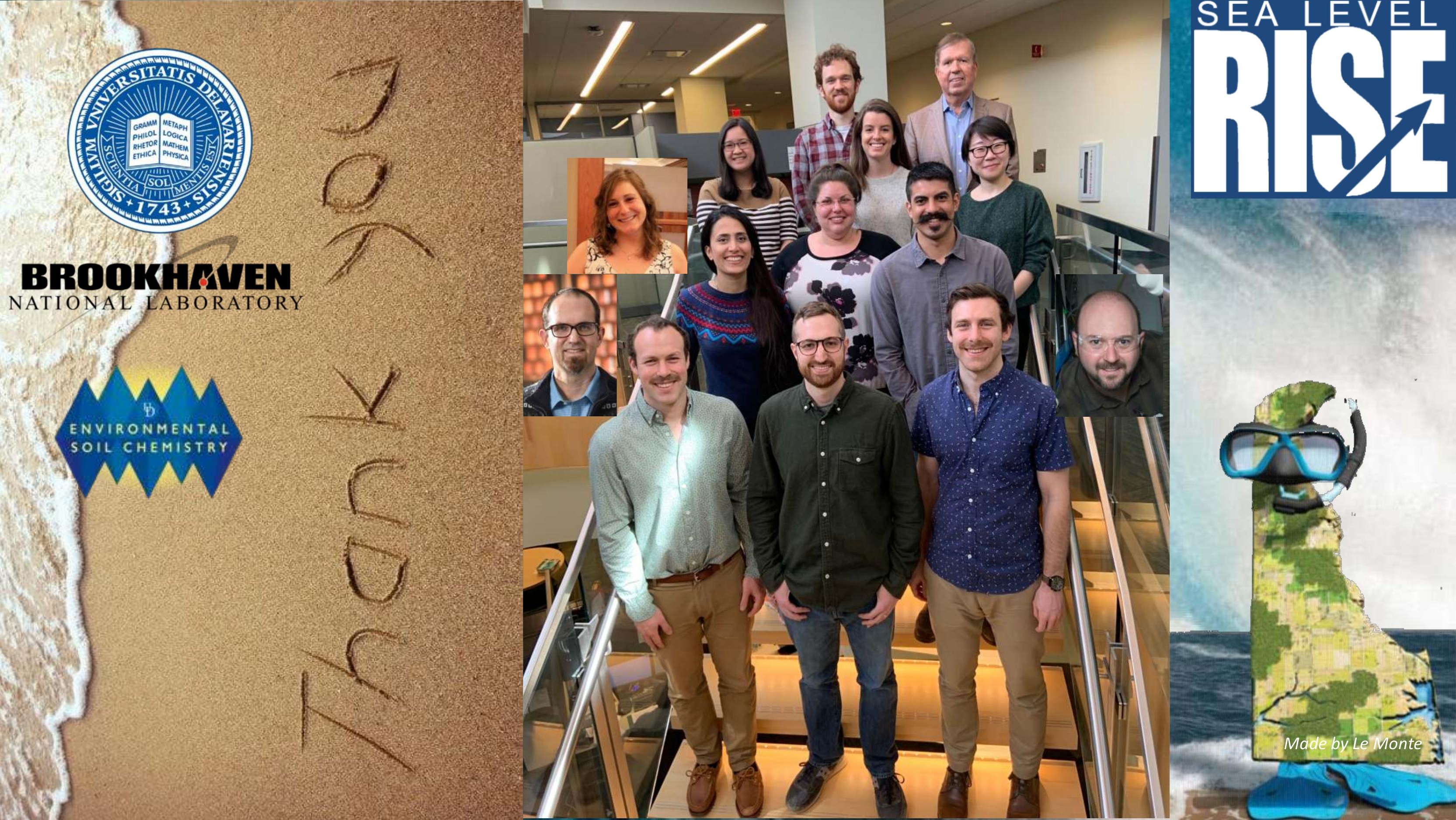

